\title{
Round Robin test on V-shape bio-imaging transfer standard for determination of the Instrument Transfer Function of 3D optical profilers
}

\section{Bermudez, Carlos}

SPIE - the international society for optics and photonics

2018-02

Bermudez , C , Artigas , R, Martinez , P , Nolvi , A , Järvinen , M , Haeggström , E \& Kassamakov , I 2018 , Round Robin test on V-shape bio-imaging transfer standard for determination of the Instrument Transfer Function of 3D optical profilers . in T G Brown , C J Cogswell \& T Wilson (eds), Three-Dimensional and Multidimensional Microscopy : Image Acquisition and Processing XXV ., UNSP 1049923 , Proceedings of SPIE , vol. 10499, SPIE - the international society for optics and photonics, Washington , Conference on Three-Dimensional and Multidimensional Microscopy: Image Acquisition and Processing XXV , San Francisco , Canada , 29/01/2018 . https://doi.org/10.1117/12.2289098

http://hdl.handle.net/10138/310901

https://doi.org/10.1117/12.2289098

unspecified

acceptedVersion

Downloaded from Helda, University of Helsinki institutional repository.

This is an electronic reprint of the original article.

This reprint may differ from the original in pagination and typographic detail.

Please cite the original version. 


\title{
Round Robin test on V-shape bio-imaging transfer standard for determination of the Instrument Transfer Function of 3D optical profilers
}

\author{
Carlos Bermudez ${ }^{\mathrm{a}}$, Roger Artigas ${ }^{\mathrm{a}}$, Pol Martinez ${ }^{\mathrm{a}}$, Anton Nolvi ${ }^{\mathrm{b}}$, Miikka Järvinen ${ }^{\mathrm{b}}$, Edward \\ Hæggström ${ }^{\mathrm{b}}$, and Ivan Kassamakov b, c* \\ ${ }^{a}$ Sensofar Tech SL, Barcelona, Spain; ${ }^{\text {buniversity of Helsinki, Helsinki, Finland; }{ }^{c} H e l s i n k i ~ I n s t i t u t e ~}$ \\ of Physics, Helsinki, Finland,
}

\begin{abstract}
A V-shape Bio-Transfer-Standard (V-BTS), developed and produced at the University of Helsinki (UH), was measured in two laboratories. In comparison to Siemens Star calibration specimens, the V-BTS performs better at high lateral frequencies close to the diffraction limit of the optical instrument. This permits determining of the Instrument Transfer Function (ITF). The V-BTS features two lipid bilayer steps that partly overlap each other at an angle of $20^{\circ}$, with an average height of $4.6 \pm 0.1 \mathrm{~nm}$. The Round Robin (RR) test aims to determine whether the V-BTS and the developed application protocol work with different optical profilers in different laboratories. First the artefact was measured at Sensofar-Tech, S.L. using an S-neox profiler working in Phase Shifting Interferometry mode. Then V-BTS was measured at UH using a custom-built Scanning White Light Interferometer. All measurements done by four different operators at the two laboratories have a range or standard deviation of $\pm 0.1 \mathrm{~nm}$ which agrees with the theoretical estimates and with measurements done using an atomic force microscope and with a surface plasmon resonance based instrument. The RR results show the applicability of the V-BTS for calibration and for ITF characterization of 3D optical profilers.
\end{abstract}

Keywords: Bio transfer standard, Optical Profiler, Coherence Scanning Interferometry, Instrument Transfer Function

\section{INTRODUCTION}

Bio-imaging, especially label-free bio-imaging, is globally large and is rapidly growing both academically and commercially. The main trends are 3D imaging, super-resolution imaging (Nobel Prize 2013), high-throughput imaging (currently hundreds of samples per microscope per day), label-free imaging (no fluorophores or stains), fresh-sample imaging (little or no sample preparation), and quantitative (metrologically traceable) imaging. For the last one there is a need for a soft transfer standard. Without traceability, measurement results are not repeatable and cannot be compared across laboratories, or even compared to results from the same laboratory obtained at different times or with different instruments. The high resolution and good repeatability of modern microscopes may give an illusion of high accuracy. Frequent calibration, instrument stability, and high resolution are all needed to have reliable and accurate measurements.

Today, measurement instruments are classified as contact and non-contact techniques. Most of the non-contact instruments provide areal measurements, but they are calibrated following the well-established procedures described in old ISO normatives for contact instruments, for instance measuring step height specimens and interpreting the results unidimensionally [1]. Nevertheless, different methods are nowadays being proposed to be able to characterize 3D profilers by means of areal 3D measurements [2-4]. This topic is currently under discussion in the context of the ISO 25178-700 [5].

ISO 25178 part 700 also discusses how the spatial resolving power of optical instruments can be described with the Instrument Transfer Function (ITF). The ITF of a topography measurement instrument is the equivalent of the optical transfer function (OTF) for a conventional microscope. The available vertical calibration specimens are made on hard substrates, such as metal, glass or silicon. Most of these calibration standards are above $1 \mu \mathrm{m}$ step height, and only some of them have less than $50 \mathrm{~nm}$ height. There have been several attempts to manufacture a commercial step height standard

*ivan.kassamakov@helsinki.fi; 
with less than $10 \mathrm{~nm}$ height. For lateral resolution calibration, different specimens are used, such a star target. However, a 2D calibration specimen is not enough to characterize the lateral resolution for metrological purposes [6].

In this paper, we propose a V-shape Bio-Transfer-Standard (BTS) that was developed and produced at the University of Helsinki (UH), Finland. To study the performance of the BTS a Round Robin test was performed. First the artefact was measured at Sensofar-Tech, S.L. using an S-neox working either in Phase Shifting Interferometry mode [7] or in Imaging Confocal Microscopy mode [8]. Then the BTS was measured at UH using a custom-built Coherence Scanning Interferometer [9]. Finally, the measurements were contrasted with an Atomic Force Microscope at the MIKES Metrology center of the VTT of Finland.

\section{METHODS}

All metrological applications rely on a calibration standard: a sample with known dimensions. The standard permits the measuring system (instrument) to be calibrated and its accuracy to be verified. To be scientifically valid, the material and dimensions of the transfer standard (TS) should closely match the properties of the actual sample that the system measures. Current transfer standards are made of metal, silicon, and other hard and technically oriented materials. The refractive index and surface roughness of such standards are very different from those of bio-samples and therefore do not offer a valid calibration for applications in the field of bio-imaging.

\subsection{Calibration by measurement standards}

Among the nine ISO specification standards regarding the measurement and characterization of surface profile, step height measurement follows the ISO 5436-1 method. The procedure is described for step height groove types, with a precisely localized step and two side locations for height referencing. The ISO procedure states to measure the width of the step $(W)$ and avoid 1/3 of the width to remove any effect of the step height walls (Fig. 1).

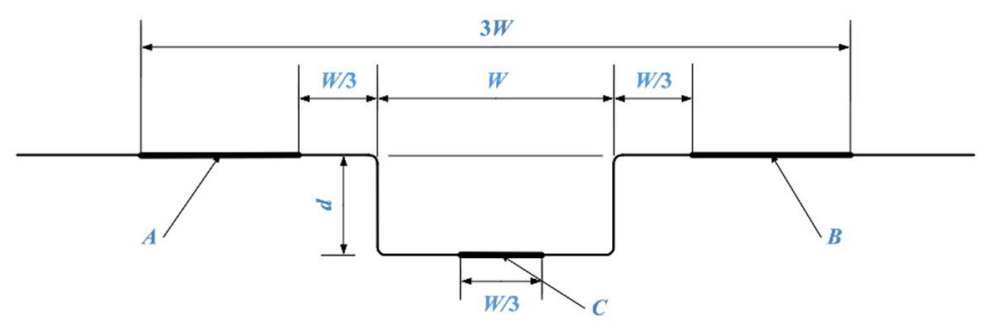

Figure 1. Step height measurement procedure described in the ISO 5436-1.

A single step height standard containing only one traceable height value is useful to calibrate the amplification coefficient of the instrument scanner, responsible at the end of the accuracy of the measurement. Successive measurements close to the calibrated value will have traceability, but this will not ensure that heights above or below the calibrated one will keep good accuracy. To ensure that, a set of several step height standards covering the desired height ranges wanted to be measured are needed for the calibration of the amplification coefficient and linearity.

The above method is currently used on 3D measuring instruments, but it does not use the benefit of areal information, but only 2D information on a cross profile. In the new ISO 25178-700 plan for surface texture standards, profile standards will develop into a sub-set of areal standards. Currently the ISO TC213 WG 16 is working on a draft of the ISO 25178-700 to describe the calibration specimens that will be intended to use for said calibration of the amplification coefficient and linearity.

\subsection{Instrument Transfer Function (ITF)}

The lateral resolution is the smallest distance between two features which can be detected. In an imaging system it is relatively simple to determine using a one-dimensional grating or ruler and give a numerical value according, for instance, 
to the Rayleigh criterion. Nevertheless, defining the resolution of a topography measuring instrument is not as straightforward. According to the ISO 25178-600, the "topographical spatial resolution" is the ability to distinguish closely spaced surface features. A practical test for defining the topographical spatial resolution is to determine the lateral period limit (spatial period of a sinusoidal profile at which the height response of an instrument falls to 50\%) of a 3D profiler through the Instrument Transfer Function, obtained from the measurement of a step-height.

As stated in the introduction, the ITF of a topography measuring instrument corresponds to the Optical Transfer Function (OTF) of a conventional microscope, assuming spatially coherent illumination. The OTF can be obtained from the inverse Fourier transform of the point spread function (PSF), which describes de response of an imaging system to a point source or point object. The PSF can be also understood as the impulse response of a focused optical system, and will contain the information of the resolution of the instrument. Determining the OTF has some difficulties, such as the necessity of measuring an infinitely small point and read the phase data, which is not always available for the average user in commercial instruments.

The equivalent of the OTF for topography measuring instruments is the ITF, which describes how the instrument would respond to an object surface having a specific spatial frequency. All the surfaces have a finite spatial frequency bandwidth, and a surface topography instrument has a finite spatial frequency response. Therefore, the ITF would depict the measured amplitude of every sinusoidal frequency on a surface, according to their bandwidth.

Several approaches have been followed to determine the ITF of an instrument. For instance, by the use of one-dimensional gratings, sinusoidal artifacts, or a 3D star (Siemens star) pattern, being this latter commercially available from NPL (National Physics Laboratory, UK) with heights down to $50 \mathrm{~nm}$ and lateral size from $0.2 \mu \mathrm{m}$ to $6 \mu \mathrm{m}$. The lateral period limit from the star can be obtained by extracting circular profiles concentric to the center of the star or by subtracting profiles extracted from the bottom and top petals of the star (Fig. 2).

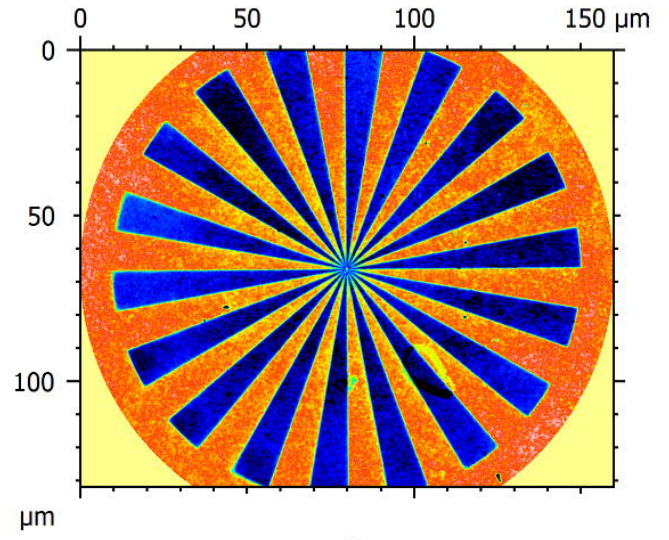

a

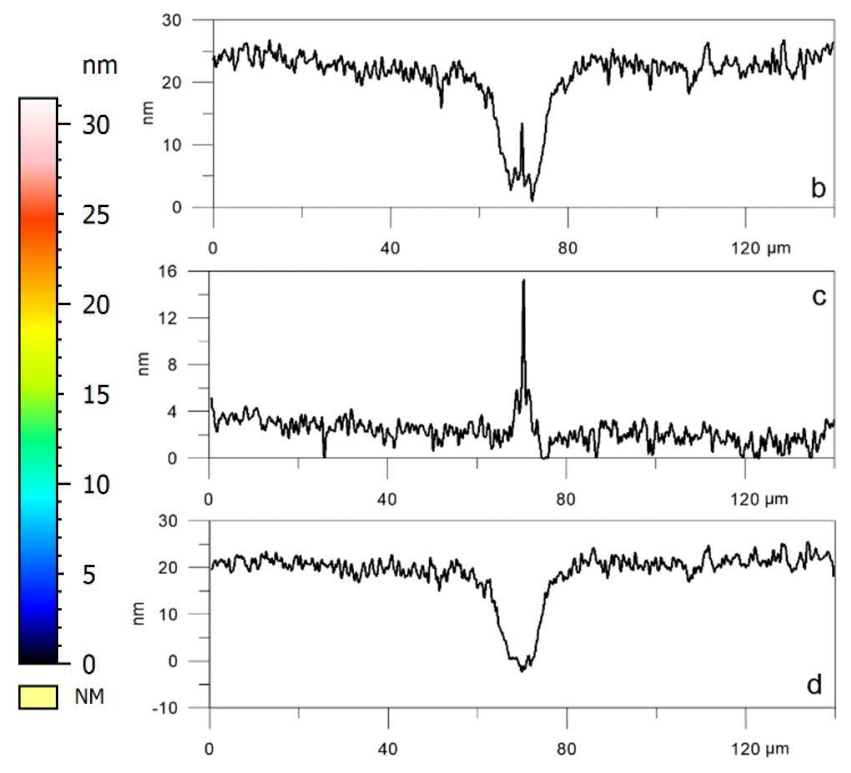

Figure 2. Star artefact measured with confocal technique, using 100X 0.9NA objective. (a) 3D measurement, (b) profile at the top of one petal (red), (c) profile at the bottom of one petal (blue), (d) former two profiles subtracted

The most relevant region of the star pattern is the central part, where the frequencies are higher. However, this is the most complex part to manufacture, as shown in the zoom view in Figure 3. Moreover, the selection of the two profiles is user dependant, and the superposition between them before the subtraction as well. Besides, in the topography is also shown that the surface has a certain waviness, adding an additional error to the measurement. 


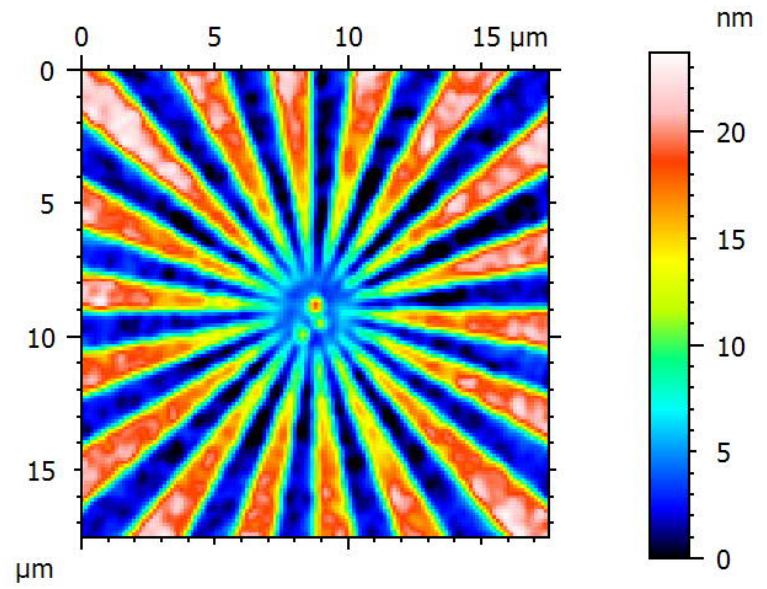

Figure 3. Detailed view of the star pattern. The center surroundings are not accurate.

Last but not least, measuring 3D specimens like the Siemens Star with an Atomic Force Microscope (AFM) is not trivial. Although they can profile better the central part of the star (laterally smaller features) than with an optical profiler, AFM resolution depends strongly on the scanning direction, making grooves not to be distinguished clearly, and measured height not corresponding to the specifications [6].

\subsection{Bio transfer standard}

Nature is 3D, and it can't be truly understood through 2D images. Existing technologies for manufacturing 3D TS rely on mechanical processes, most of the times with manually operated machines. To characterize bio-samples in a 3D and quantitative manner measurements should be calibrated both in the out-of-plane direction and in the in-plane direction. Currently existing nm-size calibration standards are unsuitable for use with bio-samples, because of phase change in light upon transmission or reflection. For true imaging a TS made by a material optically similar to the sample under investigation is necessary. To address the need for having true 3D images we introduce a TS whose dimensions are 'guaranteed' by natural self-assembly.

The BTS is based on applying Langmuir-Blodgett films (LBF) of a certain material (e.g. stearic acid) onto a desired surface (e.g. mica or glass microscopy slide) to form a flight of steps. The nature of the LBF makes every single layer equally thick, only a few nanometres tall. Partly overlapping placement (horizontal offset) of these films produces a step structure suitable for both off-line instrument calibration and for online and in-view calibration purposes. When two films are placed at a certain angle between them, V-shape, double step-height is provoked, with an extremely well defined tip (Fig. 4).

The BTS was manufactured by the Langmuir-Blodgett technique by using a KSV Minitrough (KSV Instruments, Helsinki, Finland). Briefly, a monolayer of stearic acid was first spread onto a sub-phase containing $50 \mu \mathrm{M}$ Uranylacetate (UAc) and compressed at $10 \mathrm{~mm} / \mathrm{min}$ to a surface pressure of $45 \mathrm{mN} / \mathrm{m}$. Then, 2 bilayers of the stearic acid monolayer were transferred onto a glass microscopy slide. This was achieved by intermittently immersing and withdrawing the slide into the subphase through the stearic acid monolayer 2 times at a speed of $2 \mathrm{~mm} / \mathrm{min}$, while keeping the surface pressure constant at $45 \mathrm{mN} / \mathrm{min}$ during the entire deposition process. The flight of the steps was formed during the deposition process at the three-point contact line between the microscope slide, air, and the monolayer covered subphase. The angle between the flight of steps was realized by immersing the slide to a different angle during the two bilayer deposition cycle. The thickness of similarly prepared stearic acid monolayers have been determined with a surface plasmon resonance technique to be between $2.24-2.48 \mathrm{~nm}$, which corresponds to a bilayer thickness between $4.48-4.96 \mathrm{~nm}$. 


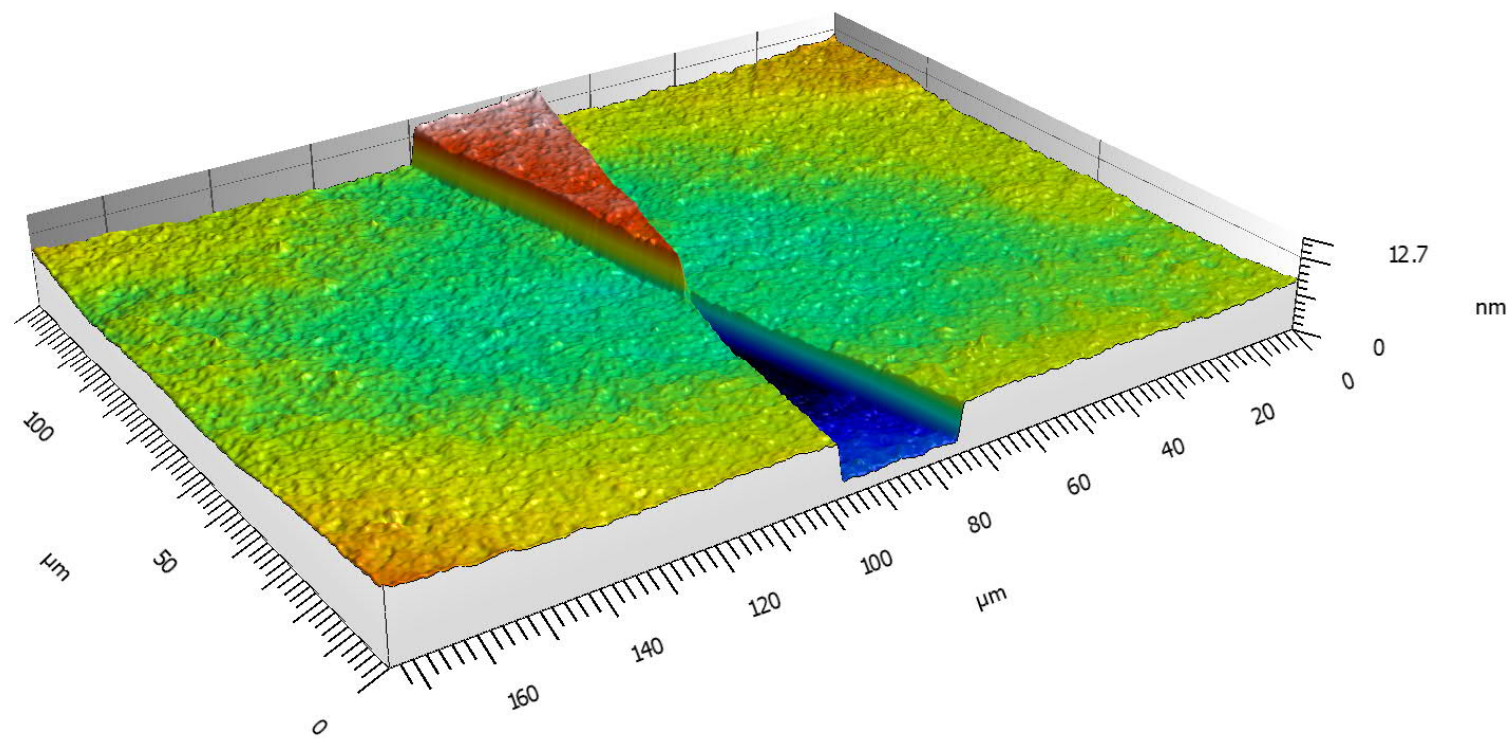

Figure 4. Langmuir-Blodgett films (LBF) based Bio-Transfer-Standard. Partly overlapping placement (angle offset) of the LBFs produces a step structure suitable for both off-line instrument calibration and for online and in-view calibration purposes.

The proposed $\mathrm{V}$-shape specimen has several advantages in comparison to traditional 2D or 3D calibration specimens. The first one, and the main reason to propose such a specimen, is that it can allow calibrating the lateral resolution not on a 2D inspection instrument, but in a 3D metrology device, because of its 3D nature. Second, it is manufactured in a flat substrate, and the two deposited layers are only some nanometre height and they are extremely repetitive, due to its molecule-based deposition [10]. The manufacturing process makes the specimen to have a curvature radius at the center around 4nm, much smaller than can be resolved by optical means. Regarding to the measurement process, as opposed to the proposed star target measurement, lateral resolution evaluation is obtained from a single profile that crosses the "V", as will be introduced in the next subsection.

\subsection{Optical measurement}

Three dimensional measurements of surfaces is an accepted technique in many fields. There are many different optical techniques that provide height sensitivity with a few $\mathrm{nm}$ of resolution. In general, optical methods are either single point or imaging detectors. Regarding imaging sensors, the most common 3D optical profilometers are focus variation, interferometers, and confocal microscopes.

Focus variation relies on the shape-from-focus technique, in which an algorithm detects high frequency details in the surface under inspection. The smallest vertical resolution that one may obtain with Focus Variation can be down to 100 $\mathrm{nm}$. Interferometers resolve height deviations below $1 \mathrm{~nm}$. If the interferometer employs a broadband spectrum light source, such as a white light LED, the interferometer is called Coherence Scanning Interferometer (CSI). The recorded signal along the optical axis is close to sinusoidal shape with its amplitude modulated by a Gaussian function with narrow width, on the order of the coherence length of the light source. The surface height is retrieved from the position of the maximum of such amplitude. The instrument noise in a CSI system is close to $1 \mathrm{~nm}$. If the interferometer features a narrow band light source, such as a monochromatic LED, the interferometer is called a Phase Shifting Interferometer (PSI). In this case, the signal recorded along the optical axis has a long amplitude modulation due to the large coherence length of the light source. In PSI systems, the phase offset for each pixel is measured resulting in a phase map of the surface. This map can be converted into a height map with the use of the mean wavelength of the light source. PSI systems achieve low instrument noise, even as low as $0.02 \mathrm{~nm}$.

Confocal systems are a trade-off between interferometers and focus variation, and are therefore the most versatile instruments in terms of application range. The optical and mechanical arrangement of a confocal microscope is complex, but it suppresses the signal that falls outside the depth of focus of the objective. The instrument noise, which depends on the numerical aperture $(N A)$ of the objective, can reach $1 \mathrm{~nm}$ for objectives with a $N A=0.95$. 
The measurement procedure to obtain the lateral resolution value with the V-Shape calibration specimen is detailed as follows. First of all, edges of the "V" are detected (Figure 5). It can be performed by image processing means, for instance with a Sobel filter. A profile coincident with the angle bisector passes through the tip of the "V".

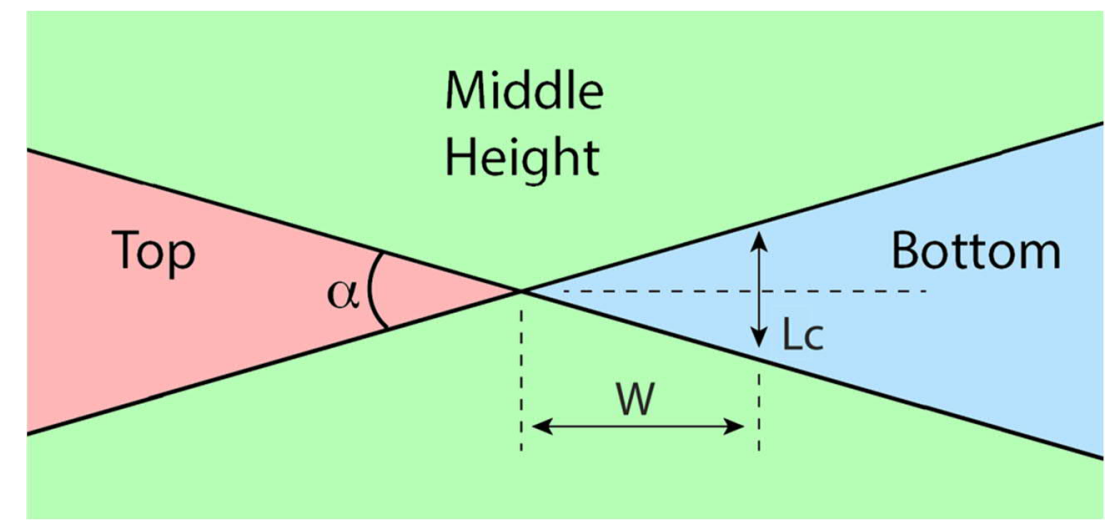

Figure 5. Geometry schematic of the V-shape specimen. $\alpha$ is the angle of the "V", and the dashed line shows its bisector angle. Three height levels are depicted: Bottom (substrate), Middle Height (only one deposition), and Top (two depositions). $W$ is the distance from the tip at which the width $\left(L_{C}\right)$ of the step is resolving $50 \%$ of the total height.

The profile that can be obtained from the angle bisector of the "V" is shown in Figure 6.

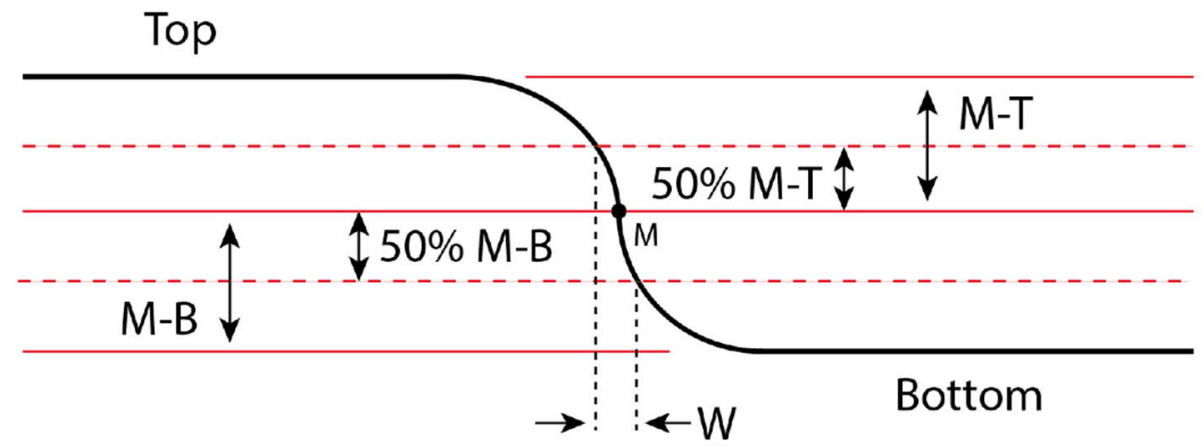

Figure 6. Profile crossing the "V" angle bisector (black). Straight red lines depict the top and the bottom area of the profile, and half the height between them. Dashed red lines are placed 50\% of the distance from the mid-height (M) to the top (T), and the same for the bottom part (M-B). $W$ indicates the distance of the points on the profile that cross the dashed lines.

In the profile, the flat areas are evaluated at a certain distance out from the "V" tip. These flat areas, top and bottom, give the maximum and minimum height values, the former corresponding to the coating and the latter to the substrate. The profile crosses the mid-height between top and bottom, which has the highest slope of the lowest frequencies in the whole profile. Along this point, the distance given by the 50\% of the height to the bottom, and the $50 \%$ of the height to the top gives the distance that can still be resolved at the $50 \%$ of the ITF.

Getting back to Figure 5, the lateral size $\mathrm{L}_{\mathrm{C}}$ that can be resolved can be calculated by:

$$
L_{C}=2 W \tan \left(\frac{\alpha}{2}\right)
$$




\section{RESULTS}

The V-BTS standard manufactured at the University of Helsinki and an NPL Siemens Star specimen have been measured with two different instruments at two different locations. At UH, a custom made white light interferometer in CSI mode was used with a 10X Linnik objective. At Sensofar Tech SL, the measurements were performed with an S-neox optical profiler in PSI mode using 4 different Mirau objectives: 10X, 20X, 50X, and 100X. Three different LED sources were used: red $(613 \mathrm{~nm})$, green $(525 \mathrm{~nm})$, and blue $(460 \mathrm{~nm})$.

Regarding to the Siemens Star evaluation, the lateral resolution results according to the method explained in subsection 2.2, are the following:

Table 1. Lateral resolution evaluation with a 3D Siemens Star, with three different wavelengths and 4 different magnifications. Between brackets, diffraction limit, according to Rayleigh's criterion.

\begin{tabular}{|c|c|c|c|}
\hline Objective & $\begin{array}{c}\text { Red }(6 \mathbf{6 1 3} \mathbf{~ n m}) \\
\boldsymbol{L}_{C}(\boldsymbol{\mu m})\end{array}$ & $\begin{array}{c}\text { Green }(\mathbf{5 2 5} \mathbf{~ n m}) \\
\boldsymbol{L}_{C}(\boldsymbol{\mu m})\end{array}$ & $\begin{array}{c}\text { Blue }(\mathbf{4 6 0} \mathbf{~ n m}) \\
\boldsymbol{L}_{C}(\boldsymbol{\mu m})\end{array}$ \\
\hline Mirau 10X 0.3NA & $1.932(1.246)$ & $1.746(1.068)$ & $2.140(0.935)$ \\
\hline Mirau 20X 0.4NA & $1.045(0.935)$ & $1.320(0.801)$ & $0.992(0.702)$ \\
\hline Mirau 50X 0.55NA & $0.629(0.680)$ & $0.552(0.582)$ & $0.634(0.510)$ \\
\hline Mirau 100X 0.7NA & $0.345(0.534)$ & $0.341(0.458)$ & $0.394(0.401)$ \\
\hline
\end{tabular}

Since the measuring instrument had only one single tube lens, it is worth noting that $10 \mathrm{X}$ and $20 \mathrm{X}$ objective are not fulfilling the Nyquist sampling criteria and thus not able to resolve diffraction limit, $1.29 \mu \mathrm{m}$ and $0.65 \mu \mathrm{m}$ respectively. Conversely, 50X and 100X objectives are diffraction limited, with samplings of $0.26 \mu \mathrm{m}$ and $0.13 \mu \mathrm{m}$ respectively.

As regards to the V-BTS, a specimen with $\alpha=22.298^{\circ}$ was measured according to the method developed in subsection 2.4. Figure 7 shows a profile of a real measurement with a 100X 0.7NA objective and green light. Distance $W$ is obtained from the crossings of the dashed lines (50\% M-B and 50\% M-T) with the profile. The rest of the measurements of $W$ and $L_{C}$ calculation is summarized in Table 2.

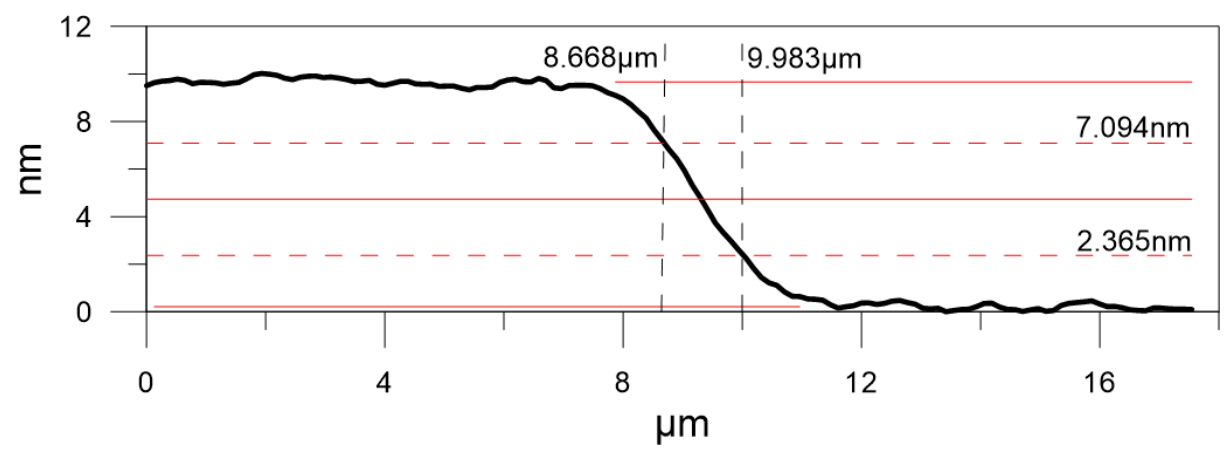

Figure 7. Profile crossing the "V" angle bisector (black). Measurement taken with the 100X 0.7NA objective and green (525 $\mathrm{nm}$ ) light. The separation between the two crossings of the profile with the red dashed lines gives the distance $W$, in this case $1.315 \mu \mathrm{m}$. 
Table 2. Lateral resolution evaluation with the V-BTS, with three different wavelengths and 4 different magnifications.

Between brackets, diffraction limit, according to Rayleigh's criterion.

\begin{tabular}{|c|c|c|c|c|c|c|}
\hline \multirow{2}{*}{ Objective } & \multicolumn{2}{|c|}{ Red $(\mathbf{6 1 3} \mathbf{~ n m})$} & \multicolumn{2}{c|}{ Green $(\mathbf{5 2 5} \mathbf{~ n m})$} & \multicolumn{2}{c|}{ Blue $(\mathbf{4 6 0} \mathbf{~ n m})$} \\
\cline { 2 - 7 } & $\boldsymbol{W}(\boldsymbol{\mu m})$ & $\boldsymbol{L}_{C}(\boldsymbol{\mu m})$ & $\boldsymbol{W}(\boldsymbol{\mu m})$ & $\boldsymbol{L}_{C}(\boldsymbol{m} \mathbf{m})$ & $\boldsymbol{W}(\boldsymbol{\mu m})$ & $\boldsymbol{L}_{C}(\boldsymbol{\mu m})$ \\
\hline Mirau 10X 0.3NA & 6.500 & $1.333(1.246)$ & 6.500 & $1.333(1.068)$ & 10.400 & $2.132(0.935)$ \\
\hline Mirau 20X 0.4NA & 3.882 & $0.796(0.935)$ & 3.233 & $0.663(0.801)$ & 4.513 & $0.925(0.702)$ \\
\hline Mirau 50X 0.55NA & 2.072 & $0.425(0.680)$ & 1.589 & $0.326(0.582)$ & 2.857 & $0.586(0.510)$ \\
\hline Mirau 100X 0.7NA & 1.424 & $0.292(0.534)$ & 1.315 & $0.270(0.458)$ & 1.977 & $0.405(0.401)$ \\
\hline
\end{tabular}

$10 \mathrm{X}$ and 20X objectives do have the internal reference mirror on a fixed position, while 50X and 100X this mirror can be adjusted axially to compensate the focus shift for different wavelengths. Due to this capability, 50X and 100X were adjusted to match specimen focus and maximum fringe contrast for every wavelength. In contrast, 10X and 20X are optimized for the green wavelength, being the red not too much far, but blue with focus shift error of several microns.

\section{DISCUSSION}

In this section results shown formerly are examined. A set of uncertainties have been identified, and the most relevant ones are reviewed in subsection 4.1. Due to the particular shape of the transfer standard presented in this paper, the angle of the "V" is of particular importance, being this examined in subsection 4.2.

\subsection{Uncertainties}

We have identified two error sources that limit the determination of the topographical resolution: spatial uncertainty due to sampling and height uncertainty. The sampling of the 3D profiler depends on the pixel size of the camera, the objective magnification, and field lens multiplier. In the case of the Sensofar S-neox profiler, the camera has a pixel size of $6.45 \mu \mathrm{m}$, and a fixed field lens of $0.5 \mathrm{X}$. Table 3 shows the resolution uncertainty dependence with spatial sampling.

Table 3. Resolution uncertainty dependence with the microscope sampling.

\begin{tabular}{|c|c|c|c|c|}
\hline & $\mathbf{1 0 X}$ & $\mathbf{2 0 X}$ & $\mathbf{5 0 X}$ & $\mathbf{1 0 0 X}$ \\
\hline Sampling $(\mu \mathrm{m})$ & 1.29 & 0.645 & 0.258 & 0.129 \\
\hline Resolution uncertainty $(\mu \mathrm{m})$ & 0.50 & 0.25 & 0.10 & 0.05 \\
\hline
\end{tabular}

On the other side, the method explained in section 2.4 requires the measurement of the step height between the substrate and the two layer deposition. This height determination needs to avoid the central region, which suffers the optical diffraction and hence the two sections used on both sides decrease in total length. The less the amount of pixels used to compute the average, the higher the uncertainty of the step height.

\subsection{Role of the angle}

The angle of the V-shape standard plays a big role for the determination of the lateral resolution of the optical profiler. We have ascertained that the optimal angle should meet at least two conditions: the smaller angle as possible, and that the "S" zone of Figure 6 to be a maximum of $1 / 3$ of the field of view of a 100X objective. The smaller the angle, the larger the "S" zone. A larger diffraction area makes the method more sensitive to the determination of the lateral resolution since the amount of pixels in the "S" area is larger. The tradeoff is on the amount of pixels used for averaging of the step height, as explained in the previous section. If we take into consideration that the field of view of a 100X is $175 \mu \mathrm{m}$, having a diffraction area of $1 / 3$ of that field $(58 \mu \mathrm{m})$, and staying at least three times further away from the diffraction limit (about 
$1 \mu \mathrm{m}$ for the 100X objective), the optimal angle is about $1^{\circ}$. If we repeat the same calculation for the 10X objective it will be about $0.5^{\circ}$. In this paper we used a specimen manufactured with $22.298^{\circ}$ angle. Despite this is not the optimal angle we proved that it is still sufficient for the characterization of the lateral resolution.

\section{CONCLUSION}

In this paper, a new 3D, V-shaped lateral resolution specimen for the determination of the Instrument Transfer Function (ITF) has been presented and evaluated. Compared to traditional specimens, the V Bio-Transfer-Standard (V-BTS) is only a few nanometres height and extremely repeatable to manufacture. Measurements have been performed with Coherence Scanning Interferometry (CSI) with three different wavelengths and 4 different Mirau objectives. Results show a clear tendency below Rayleigh's criterion both for the Siemens Star and for the V-BTS except for blue light with low magnification $(10 \mathrm{X}, 20 \mathrm{X})$ in the latter, which appears to be counterproductive. The source of uncertainties have been studied, with special attention to the optimal "V" angle. This allows the transfer standard to be used for calibration of other optical or near-field 3D devices.

\section{REFERENCES}

[1] ISO 5436 part 1, Geometrical Product Specification (GPS) - Surface Texture: Profile Method - Measurement Standards - Material Measures. International Organization of Standardization (2000)

[2] ISO 25178 part 70, Geometrical Product Specification (GPS) - Surface Texture: Areal - Part 70: Material Measures. International Organization for Standardization (2014)

[3] Giusca, C. L., Leach, R. K., and Helery, F., "Calibration of the scales of areal surface topography measuring instruments: part 2. Amplification, linearity and squareness, " Measurement Science and Technology, 2012, Vol. 23, Num. 6, 065005 (2012)

[4] de Groot, P. and Fitzgerald, D., "Measurement, certification and use of step-height calibration specimens in optical metrology," Proc. of SPIE Vol. 10329 (2017)

[5] ISO/CD 25178 part 700, Geometrical Product Specification (GPS) - Surface Texture: Areal - Part 700: Calibration of Non-contact Instruments. International Organization for Standardization (2014)

[6] Weckenmann, A., Tan, Ö., Hoffmann, J., and Sun, Z., " Practice-oriented evaluation of lateral resolution for microand nanometre measurement techniques," Measurement Science and Technology 20(6), 065103 (2009)

[7] Leach R., [Optical Measurement of Surface Topography], Springer Berlin Heidelberg (2011)

[8] Artigas R., Laguarta F., and Cadevall C., "Dual-technology optical sensor head for 3D surface shape measurements on the micro and nano-scales," SPIE Vol. 5457, 166 (2004)

[9] Sandler N., Kassamakov I., Ehlers H., Genina N., Ylitalo T. and Haeggström E., "Rapid interferometric imaging of printed drug laden multilayer structures," Scientific Reports 4, 4020 (2014)

[10] Nolvi, A., Viitala, T., García Pérez, A., Sandler, N., Hæggström, E., Bermudez, C., Artigas, R., Kassamakov, I., "Round Robin test on bio-imaging transfer standard for 3D optical profilers," Proc. SPIE 10110, Photonic Instrumentation Engineering IV, 101100M (2017) 